TRANSACTIONS OF THE

AMERICAN MATHEMATICAL SOCIETY

Volume 351, Number 3, March 1999, Pages 997-1014

S 0002-9947(99)02201-1

\title{
COMPATIBLE COMPLEX STRUCTURES ON ALMOST QUATERNIONIC MANIFOLDS
}

\author{
D. V. ALEKSEEVSKY, S. MARCHIAFAVA, AND M. PONTECORVO
}

\begin{abstract}
On an almost quaternionic manifold $\left(M^{4 n}, Q\right)$ we study the integrability of almost complex structures which are compatible with the almost quaternionic structure $Q$. If $n \geq 2$, we prove that the existence of two compatible complex structures $I_{1}, I_{2} \neq \pm I_{1}$ forces $\left(M^{4 n}, Q\right)$ to be quaternionic. If $n=1$, that is $\left(M^{4}, Q\right)=\left(M^{4},[g], o r\right)$ is an oriented conformal 4-manifold, we prove a maximum principle for the angle function $\left\langle I_{1}, I_{2}\right\rangle$ of two compatible complex structures and deduce an application to anti-self-dual manifolds.

By considering the special class of Oproiu connections we prove the existence of a well defined almost complex structure $\mathbb{I}$ on the twistor space $Z$ of an almost quaternionic manifold $\left(M^{4 n}, Q\right)$ and show that $\mathbb{I}$ is a complex structure if and only if $Q$ is quaternionic. This is a natural generalization of the Penrose twistor constructions.
\end{abstract}

\section{INTRODUCTION AND MAIN RESUlTS}

An almost hypercomplex structure on a $4 n$-dimensional manifold $M$ is a triple $H=\left(J_{\alpha}\right), \alpha=1,2,3$, of almost complex structures $J_{\alpha}: T M \rightarrow T M$ satisfying the quaternionic identities $J_{\alpha}^{2}=-i d$ and $J_{1} J_{2}=-J_{2} J_{1}=J_{3}$. When each $J_{\alpha}$ is a complex structure $H$ is said to be a hypercomplex structure on $M$.

An almost quaternionic structure on $M$ is a rank-3 subbundle $Q \subset \operatorname{End}(T M)$ which is locally spanned by almost hypercomplex structures $H=\left(J_{\alpha}\right)$; such a locally defined triple $H$ will be called an admissible basis of $Q$ and we will write $Q=\langle H\rangle$. We will also say that $Q$ is a quaternionic structure when there is a torsionless connection $\nabla$ on $T M$ preserving $Q$-i.e. $\nabla_{X} \sigma \in \Gamma(Q)$ for all vector fields $X$ and smooth sections $\sigma \in \Gamma(Q)$. For short we will also say that $\left(M^{4 n}, Q\right)$ is a (almost) quaternionic manifold and $\nabla$ is a (almost) quaternionic connection. For $n=1$ an almost quaternionic structure is the same as an oriented conformal structure and it turns out to be quaternionic always; however we will consider the four-dimensional case only in $\S 5$. When $n \geq 2$ instead, the existence of a torsionless connection is a fairly strong condition on $\left(M^{4 n}, Q\right)$ which is equivalent to the 1 integrability of the associated $G L(n, \mathbb{H}) S p(1)$-structure.

When $n \geq 2$ the Riemannian version of quaternionic geometry is the notion of a quaternion-Kähler manifold $\left(M^{4 n}, Q, g\right)$ where $Q$ is a quaternionic structure

Received by the editors December 14, 1996.

1991 Mathematics Subject Classification. Primary 53C10, 32C10.

Work done under the program of G.N.S.A.G.A. of C.N.R. and partially supported by M.U.R.S.T. (Italy) and E.S.I. (Vienna). 
invariant under the Levi-Civita connection of $g$. It is equivalent to say that the holonomy group of $g$ is contained in $S p(n) S p(1)$. We will not consider the quaternionKähler case here and leave the study of almost complex structures on such manifolds and on Riemannian four-manifolds to a forthcoming paper.

A first consequence of the definition of an almost quaternionic manifold is that the bundle $Q$ has structure group $S U(2) / \mathbb{Z}_{2}=S O(3)$. We then have a natural inner product $\langle$,$\rangle on Q$ by taking each admissible basis $H=\left(J_{\alpha}\right), \alpha=1,2,3$, to be an orthonormal basis. The twistor space $Z$ of $\left(M^{4 n}, Q\right)$ is defined to be the unit sphere bundle of $(Q,\langle\rangle$,$) ; the projection t: Z \rightarrow M$ is called the twistor fibration and for $p \in M$ a fiber $t^{-1}(p) \cong S^{2}$ is called a twistor fiber. A local section $I: M \rightarrow Z$ of the twistor fibration is a field of endomorphisms of the form $I=\sum_{\alpha=1}^{3} c_{\alpha} J_{\alpha}$ where $c_{\alpha}$ are smooth functions satisfying $\sum_{\alpha=1}^{3} c_{\alpha}^{2}=1$. Therefore $I^{2}=-i d$, that is, $I$ is an almost complex structure. We will say that an almost complex structure on (an open subset of) $M$ is compatible with the almost quaternionic structure $Q$ if it is a (local) section of $Z$.

Let us also recall that the existence of compatible complex structures on quaternionic manifolds $\left(M^{4 n}, Q\right)$ was investigated in [P1] while admissible almost hypercomplex structures were considered in $[\mathrm{AM}]$.

In the present paper we study the relationships between the 1-integrability of the almost quaternionic structure $Q$ and the existence of compatible complex structures. It is well known, and we also provide a lot of evidence for this fact, that quaternionic manifolds are a good analogue of anti-self-dual conformal four-manifolds in higher dimensions. For example, they have a very similar twistor theory and they can also be characterized in terms of existence of sufficiently many compatible complex structures. However there are also some differences. It is known that any Riemannian four-manifold with three compatible complex structures must be antiself-dual [S4], but there exist non anti-self-dual compact conformal four-manifolds with exactly two compatible complex structures $[\mathrm{K}]$.

On the other hand we prove in $\S 2$ that if $n \geq 2$ an almost quaternionic manifold $\left(M^{4 n}, Q\right)$ with two compatible complex structures $I_{1} \neq \pm I_{2}$ is necessarily quaternionic. A consequence is that $I_{1}$ and $I_{2}$ form a constant angle - i.e. the inner product $\left\langle I_{1}, I_{2}\right\rangle$ is a constant function - if and only if $Q=\langle H\rangle$ where $H$ is a globally defined hypercomplex structure generated by $I_{1}$ and $I_{2}$, over the real numbers $\mathbb{R}$, see Remark 2.2. This last result was known to Grantcharov [Gr] in dimension 4 and has also been proved independently by Gauduchon [G3] in any dimension.

In $\S 3$ we develop the twistor theory of almost quaternionic manifolds by considering a special class of $Q$-preserving connections called Oproiu connections [AM]. Such connections all have the same torsion and can be used to define a canonical almost complex structure on the twistor space of $\left(M^{4 n}, Q\right)$. This is a natural generalization of the twistor theory of [AHS] for $n=1$ and of [S1], [S3] for $n \geq 2$. In particular we show that for $n \geq 2$ the twistor space is a complex manifold if and only if $Q$ is a quaternionic structure.

In $\S 4$ we collect our main results and show that an almost quaternionic manifold $\left(M^{4 n}, Q\right)$ with $n \geq 2$ is quaternionic if and only if it locally admits an abundance of compatible complex structures or if and only if there locally exist two compatible complex structures. To show that our result cannot be improved, we conclude $\S 4$ with examples of almost quaternionic manifolds with a compatible complex structure which is unique even locally. 
In $\S 5$ we concentrate on the four-dimensional case and prove a "maximum principle" for the angle function $a=\left\langle I_{1}, I_{2}\right\rangle$. In particular we show that when $\left(M^{4}, g\right.$, or $)$ is compact oriented and anti-self-dual the non-constant map $a=\left\langle I_{1}, I_{2}\right\rangle: M \rightarrow$ $[-1,1]$ is surjective. This result has been used to give a classification of such Riemannian four-manifolds admitting two compatible complex structures [P2].

\section{Almost quaternionic structures}

Let $\left(M^{4 n}, Q\right)$ be an almost quaternionic manifold. In this section we consider the situation when there exist two compatible (almost) complex structures $I_{1} \neq \pm I_{2}$ which are globally defined on $M$. We can always choose a local admissible basis $H=\left(J_{\alpha}\right)$ of $Q$ such that $I_{1}=J_{1}$ and $I_{2}=a J_{1}+b J_{2}$ with $a^{2}+b^{2}=1$. We then have $\left\langle I_{1}, I_{2}\right\rangle=a$ and we will be interested in the (globally defined) "angle" function $a: M \rightarrow[-1,1]$ given by $a(p)=\left\langle I_{1}, I_{2}\right\rangle(p)$. Many of the simple algebraic properties of $I_{1}$ and $I_{2}$ can be expressed in terms of this function as explained below.

Remarks 2.1. (1) $a(p)= \pm 1$ if and only if $I_{1}= \pm I_{2}$ at $p \in M$, or if and only if they commute at $p$. Notice that by assumption $a$ is not identically equal to \pm 1 .

(2) The anti-commutator $\left\{I_{1}, I_{2}\right\}:=I_{1} I_{2}+I_{2} I_{1}$ satisfies the following identity:

$$
\left\{I_{1}, I_{2}\right\}=-a i d+b J_{3}-a i d-b J_{3}=-2 a i d ;
$$

therefore $a(p)=0$ if and only if $I_{1}$ and $I_{2}$ anti-commute at $p$. The smooth function $a: M \rightarrow[-1,1]$ measures the cosine of the angle between $I_{1}$ and $I_{2}$ and will play a fundamental role in what follows.

(3) More generally, it is possible to show [T1] that on any manifold $M$ two almost complex structures satisfying the equation

$$
\left\{I_{1}, I_{2}\right\}=-2 \text { a id }
$$

with strict inequality $|a|<1$ always span an almost hypercomplex structure on $M$.

(4) The commutator $\left[I_{1}, I_{2}\right]:=I_{1} I_{2}-I_{2} I_{1}=2 b J_{3}$ belongs to $Q$ and therefore at each point where $I_{1} \neq \pm I_{2}$ we have that $J_{3}= \pm \frac{1}{2}\left(1-a^{2}\right)^{-\frac{1}{2}}\left[I_{1}, I_{2}\right]$ is the only compatible almost complex structure (up to sign) which is orthogonal to the 2-plane spanned by $I_{1}$ and $I_{2}$.

(5) If $t r$ denotes the trace of an endomorphism of $T_{p} M$, then the function $\operatorname{tr}\left(I_{1} I_{2}\right)=\frac{1}{2} \operatorname{tr}\left\{I_{1}, I_{2}\right\}=-a \operatorname{tr}(i d)=-4 n a$. Therefore the scalar product $a=$ $\left\langle I_{1}, I_{2}\right\rangle=-\frac{1}{4 n} \operatorname{tr}\left(I_{1} I_{2}\right)$.

(6) A Riemannian metric $g$ on $\left(M^{4 n}, Q\right)$ is said to be $Q$-Hermitian if it is Hermitian with respect to every compatible almost complex structure; it is not difficult to see that such metrics always exist. Notice that for any $Q$-Hermitian metric $g$ and $g$-orthonormal frame $E_{1}, \ldots, E_{4 n}$ the following relation holds:

$$
\begin{aligned}
g\left(I_{1}, I_{2}\right) & =-\sum_{r=1}^{4 n} g\left(I_{1}\left(E_{r}\right), I_{2}\left(E_{r}\right)\right)=-\sum_{r=1}^{4 n} g\left(E_{r}, I_{1} I_{2}\left(E_{r}\right)\right) \\
& =-\operatorname{tr}\left(I_{1} I_{2}\right)=4 n\left\langle I_{1}, I_{2}\right\rangle=4 n a .
\end{aligned}
$$

A similar situation has been studied by Tricerri in [T1] and [T2] where he considered a manifold $M$ equipped with two almost complex structures $I_{1}$ and $I_{2}$ which span a subalgebra $\mathcal{S}$ of $\operatorname{End}(T M)$ of real dimension 4 and proved that there is a Riemannian metric $g$ which is Hermitian with respect to $I_{1}$ and $I_{2}$ if and only if $I_{1}$ and $I_{2}$ commute or else satisfy the algebraic condition that $I_{1} I_{2}+I_{2} I_{1}=-2 \mathrm{a} i d$ 
for some function $a: M \rightarrow(-1,1)$; he also showed that this is equivalent to the condition that $\mathcal{S}$ is isomorphic to the algebra of quaternions $\mathbb{H}$. He also constructed an almost hypercomplex structure on the total space of the tangent bundle of any almost complex manifold.

Throughout this section we will assume that $I_{1}$ and $I_{2}$ are compatible complex structures on an almost quaternionic manifold $\left(M^{4 n}, Q\right)$ with $n \geq 2$ and deduce that $Q$ is in fact a quaternionic structure. We start by recalling the following "rigidity" result which holds for any two complex structures compatible with a fixed Riemannian metric and orientation.

Remark 2.2. It was shown in [P2, Proposition 1.3] that two different complex structures $I_{1}, I_{2}$ which are compatible with the same Riemannian metric cannot have a contact of infinite order; therefore $I_{1} \neq \pm I_{2}$-i.e. $a^{2} \neq 1$-in a dense open set $\mathcal{A} \subset M$. In what follows it will be useful to notice that we can define an admissible basis $H=\left(J_{\alpha}\right)$ of $Q$ on $\mathcal{A}$ by the following formulas:

$$
I_{1}=J_{1}, \quad I_{2}=a J_{1}+b J_{2} \quad \text { with } \quad a^{2}+b^{2}=1 \quad \text { and } \quad J_{3}=J_{1} J_{2} .
$$

When $a$ and $b$ are constant and $H=\left(J_{\alpha}\right)$ is actually hypercomplex, we will say that $I_{1}$ and $I_{2}$ generate a hypercomplex structure, over $\mathbb{R}$.

We start by recalling some definitions and preliminary results on the relation between hypercomplex and quaternionic structures.

Let $H=\left(J_{\alpha}\right)$ be an almost hypercomplex structure on $M$. The structure tensor of $H$ is defined as

$$
T^{H}=\frac{1}{12} \sum_{\alpha=1}^{3}\left[\left[J_{\alpha}, J_{\alpha}\right]\right]
$$

where the Nijenhuis bracket $[[A, B]]$ of two endomorphisms is defined in terms of the Lie bracket of vector fields in the following way:

$$
\begin{aligned}
& {[[A, B]](X, Y)=[A X, B Y]-A[B X, Y]-B[X, A Y]+[B X, A Y]} \\
& -B[A X, Y]-A[X, B Y]+(A B+B A)[X, Y]
\end{aligned}
$$

for any vector fields $X$ and $Y$.

The Nijenhuis bracket of an almost complex structure $J$ is of particular importance because, by the Newlander-Niremberg Theorem, $[[J, J]]=0$ is a necessary and sufficient condition for the integrability of $J$.

For the almost hypercomplex structure $H=\left(J_{\alpha}\right)$ there exists a unique linear connection $\nabla^{H}$ which preserves $H$, that is, $\nabla^{H} J_{\alpha}=0, \alpha=1,2,3$, and whose torsion tensor equals $T^{H} . \nabla^{H}$ is called the Obata connection of $H$ (see for example [AM, p. 37]) and it is known that the torsion of $\nabla^{H}$ vanishes, $T^{H}=0$, if and only if $H$ is hypercomplex.

Setting $T_{X}^{H} Y:=T^{H}(X, Y)$ we refer to $[\mathrm{AM}]$ for the following facts:

$$
T_{X}^{H} Y=\sum_{\alpha=1}^{3} J_{\alpha} T_{X}^{H} J_{\alpha} Y
$$

and

$$
\sum_{\alpha=1}^{3} \tau_{\alpha}^{H} \circ J_{\alpha}=0
$$


where

$$
\tau_{\alpha}^{H}(X)=\frac{1}{4 n-2} \operatorname{tr}\left(J_{\alpha} T_{X}^{H}\right)
$$

are the structure 1-forms of $H$.

The structure tensor $T^{Q}$ of an almost quaternionic structure $Q$ which is locally generated by $H=\left(J_{\alpha}\right)$ is given by

$$
T^{Q}=T^{H}+\sum_{\alpha=1}^{3} \partial\left(\tau_{\alpha}^{H} \otimes J_{\alpha}\right)
$$

where $\partial$ denotes the operator of alternation.

Notice that $T^{Q}$ depends only on $Q$ and furthermore $T^{Q}=0$ if and only if $Q$ is a quaternionic structure - i.e. there is a torsionless connection preserving $Q$. This condition is always satisfied when $n=1[\mathrm{AM}]$. When $n \geq 2$, a necessary and sufficient condition for the vanishing of the tensor $T^{Q}$ is the following.

Proposition 2.3. Assume $n \geq 2$. Then $Q$ is quaternionic if and only if in a neighborhood of any point there exists a local admissible basis $H=\left(J_{\alpha}\right)$ such that the structure tensor $T^{H}$ has the form

$$
T^{H}=-\partial\left(\sum_{\alpha} a_{\alpha} \otimes J_{\alpha}+c \otimes I d\right)
$$

where $a_{\alpha}(\alpha=1,2,3)$ and $c$ are 1-forms.

Proof. It is straightforward to show that identities (2.2) and (2.3) imply $c=0$ and $\sum_{\alpha} a_{\alpha} \circ J_{\alpha}=0$. Then

$(\alpha=1,2,3)$

$$
\operatorname{tr}\left(J_{\alpha} T_{X}^{H}\right)=(4 n-2) a_{\alpha}(x)
$$

and the conclusion follows by comparing with (2.4).

We are now ready to prove the main result of the section which is in surprising contrast with the four-dimensional case [S4], [K]; see also 4.2 and 5.8.

Theorem 2.4. Let $Q$ be an almost quaternionic structure on $M^{4 n}$ with $n \geq 2$. If there exist two compatible complex structures $I_{1} \neq \pm I_{2}$, then $Q$ is a quaternionic structure.

Proof. Let $H=\left(J_{\alpha}\right)$ be the almost hypercomplex basis (2.1) defined on the open dense set $\mathcal{A}$ where $b^{2}=1-a^{2} \neq 0$ (see Remark 2.2). A straightforward calculation shows that

$$
\begin{gathered}
{\left[\left[I_{2}, I_{2}\right]\right](X, Y)=a^{2}\left[\left[J_{1}, J_{1}\right]\right](X, Y)+b^{2}\left[\left[J_{2}, J_{2}\right]\right](X, Y)+2 a b\left[\left[J_{1}, J_{2}\right]\right](X, Y)} \\
+2\left\{-\left(I_{2} Y \cdot a\right) J_{1} X+\left(I_{2} X \cdot a\right) J_{1} Y-\left(I_{2} Y \cdot b\right) J_{2} X+\left(I_{2} X \cdot b\right) J_{2} Y\right. \\
\left.+(a(Y \cdot b)-b(Y \cdot a)) J_{3} X-(a(X \cdot b)-b(X \cdot a)) J_{3} Y\right\} .
\end{gathered}
$$

By continuity it suffices to show that the structure tensor $T^{Q}=0$ on $\mathcal{A}$; in other words it suffices to show that $T^{H}$ satisfies the equation of Proposition 2.3. For this purpose in the calculations of the present proof we are allowed to neglect terms of the form

$$
\partial\left(\sum_{\alpha} a_{\alpha} \otimes J_{\alpha}+c \otimes I d\right)
$$


and by $\equiv$ we will mean that two expressions are equal up to terms of the above specified type. For example the above equation is replaced by the following equivalence:

$$
\left[\left[I_{2}, I_{2}\right]\right](X, Y) \equiv a^{2}\left[\left[J_{1}, J_{1}\right]\right](X, Y)+b^{2}\left[\left[J_{2}, J_{2}\right]\right](X, Y)+2 a b\left[\left[J_{1}, J_{2}\right]\right](X, Y) .
$$

In order to show that $T^{H} \equiv 0$ we will use the integrability of $I_{1}=J_{1}$ and $I_{2}$ to prove that $\left[\left[J_{2}, J_{2}\right]\right] \equiv\left[\left[J_{3}, J_{3}\right]\right] \equiv 0$. To see this we will start by computing $\left[\left[J_{1}, J_{2}\right]\right]$. Notice that $\left[\left[J_{2}, J_{2}\right]\right] \equiv 0$ certainly holds at all points where $a=0$ because of $(2.5)$ and the fact that $I_{2}$ is integrable. Therefore in the following computation it is enough to assume that $a b \neq 0$.

From $[\mathrm{AM},(3.4 .1)]$ we have that

$$
\begin{aligned}
{\left[\left[J_{1}, J_{2}\right]\right](X, Y)=} & J_{1} T_{X}^{H} J_{2} Y+J_{2} T_{X}^{H} J_{1} Y+J_{1} T_{J_{2} X}^{H} Y+J_{2} T_{J_{1} X}^{H} Y \\
& -T_{J_{1} X}^{H} J_{2} Y-T_{J_{2} X}^{H} J_{1} Y .
\end{aligned}
$$

Then

$$
\begin{aligned}
& 12\left[\left[J_{1}, J_{2}\right]\right](X, Y)= \\
& J_{1}\left[\left[J_{2}, J_{2}\right]\right]\left(X, J_{2} Y\right)+J_{1}\left[\left[J_{3}, J_{3}\right]\right]\left(X, J_{2} Y\right)+J_{2}\left[\left[J_{2}, J_{2}\right]\right]\left(X, J_{1} Y\right)+ \\
& J_{2}\left[\left[J_{3}, J_{3}\right]\right]\left(X, J_{1} Y\right)+J_{1}\left[\left[J_{2}, J_{2}\right]\right]\left(J_{2} X, Y\right)+J_{1}\left[\left[J_{3}, J_{3}\right]\right]\left(J_{2} X, Y\right)+ \\
& J_{2}\left[\left[J_{2}, J_{2}\right]\right]\left(J_{1} X, Y\right)+J_{2}\left[\left[J_{3}, J_{3}\right]\right]\left(J_{1} X, Y\right)-\left[\left[J_{2}, J_{2}\right]\right]\left(J_{1} X, J_{2} Y\right)- \\
& {\left[\left[J_{3}, J_{3}\right]\right]\left(J_{1} X, J_{2} Y\right)-\left[\left[J_{2}, J_{2}\right]\right]\left(J_{2} X, J_{1} Y\right)-\left[\left[J_{3}, J_{3}\right]\right]\left(J_{2} X, J_{1} Y\right) .}
\end{aligned}
$$

Now we recall the following formula which was established in [AM, (3.4.4)]:

$$
\begin{aligned}
& 2\left[\left[J_{3}, J_{3}\right]\right](X, Y)=\left[\left[J_{1}, J_{1}\right]\right](X, Y)-J_{2}\left[\left[J_{1}, J_{1}\right]\right]\left(J_{2} X, Y\right)- \\
& \quad J_{2}\left[\left[J_{1}, J_{1}\right]\right]\left(X, J_{2} Y\right)+\left[\left[J_{1}, J_{1}\right]\right]\left(J_{2} X, J_{2} Y\right)+\left[\left[J_{2}, J_{2}\right]\right](X, Y)- \\
& \quad J_{1}\left[\left[J_{2}, J_{2}\right]\right]\left(J_{1} X, Y\right)-J_{1}\left[\left[J_{2}, J_{2}\right]\right]\left(X, J_{1} Y\right)+\left[\left[J_{2}, J_{2}\right]\right]\left(J_{1} X, J_{1} Y\right) .
\end{aligned}
$$

Because $J_{1}$ is integrable we obtain

$$
\begin{aligned}
& 24\left[\left[J_{1}, J_{2}\right]\right](X, Y)= \\
& 2\left\{J_{1}\left[\left[J_{2}, J_{2}\right]\right]\left(X, J_{2} Y\right)+J_{2}\left[\left[J_{2}, J_{2}\right]\right]\left(X, J_{1} Y\right)+J_{1}\left[\left[J_{2}, J_{2}\right]\right]\left(J_{2} X, Y\right)+\right. \\
& \left.\quad J_{2}\left[\left[J_{2}, J_{2}\right]\right]\left(J_{1} X, Y\right)-\left[\left[J_{2}, J_{2}\right]\right]\left(J_{1} X, J_{2} Y\right)-\left[\left[J_{2}, J_{2}\right]\right]\left(J_{2} X, J_{1} Y\right)\right\}+ \\
& J_{1}\left[\left[J_{2}, J_{2}\right]\right]\left(X, J_{2} Y\right)+\left[\left[J_{2}, J_{2}\right]\right]\left(J_{1} X, J_{2} Y\right)+\left[\left[J_{2}, J_{2}\right]\right]\left(X, J_{3} Y\right)+J_{1}\left[\left[J_{2}, J_{2}\right]\right]\left(J_{1} X, J_{3} Y\right)+ \\
& J_{2}\left[\left[J_{2}, J_{2}\right]\right]\left(X, J_{1} Y\right)+J_{3}\left[\left[J_{2}, J_{2}\right]\right]\left(J_{1} X, J_{1} Y\right)-J_{3}\left[\left[J_{2}, J_{2}\right]\right](X, Y)-J_{2}\left[\left[J_{2}, J_{2}\right]\right]\left(J_{1} X, Y\right)+ \\
& J_{1}\left[\left[J_{2}, J_{2}\right]\right]\left(J_{2} X, Y\right)+\left[\left[J_{2}, J_{2}\right]\right]\left(J_{3} X, Y\right)+\left[\left[J_{2}, J_{2}\right]\right]\left(J_{2} X, J_{1} Y\right)+J_{1}\left[\left[J_{2}, J_{2}\right]\right]\left(J_{3} X, J_{1} Y\right)+ \\
& J_{2}\left[\left[J_{2}, J_{2}\right]\right]\left(J_{1} X, Y\right)-J_{3}\left[\left[J_{2}, J_{2}\right]\right](X, Y)+J_{3}\left[\left[J_{2}, J_{2}\right]\right]\left(J_{1} X, J_{1} Y\right)-J_{2}\left[\left[J_{2}, J_{2}\right]\right]\left(X, J_{1} Y\right)- \\
& {\left[\left[J_{2}, J_{2}\right]\right]\left(J_{1} X, J_{2} Y\right)-J_{1}\left[\left[J_{2}, J_{2}\right]\right]\left(X, J_{2} Y\right)+J_{1}\left[\left[J_{2}, J_{2}\right]\right]\left(J_{1} X, J_{3} Y\right)+\left[\left[J_{2}, J_{2}\right]\right]\left(X, J_{3} Y\right)-} \\
& {\left[\left[J_{2}, J_{2}\right]\right]\left(J_{2} X, J_{1} Y\right)+J_{1}\left[\left[J_{2}, J_{2}\right]\right]\left(J_{3} X, J_{1} Y\right)-J_{1}\left[\left[J_{2}, J_{2}\right]\right]\left(J_{2} X, Y\right)+\left[\left[J_{2}, J_{2}\right]\right]\left(J_{3} X, Y\right) .}
\end{aligned}
$$

Then, by taking into account the following identities for the Nijenhuis tensor of the almost-complex structure $J_{2}$, that is

$$
\begin{gathered}
{\left[\left[J_{2}, J_{2}\right]\right]\left(J_{2} X, Y\right)=\left[\left[J_{2}, J_{2}\right]\right]\left(X, J_{2} Y\right)=-J_{2}\left[\left[J_{2}, J_{2}\right]\right](X, Y),} \\
{\left[\left[J_{2}, J_{2}\right]\right]\left(J_{2} X, J_{2} Y\right)=-\left[\left[J_{2}, J_{2}\right]\right](X, Y),}
\end{gathered}
$$

we conclude that

$$
\begin{aligned}
4\left[\left[J_{1}, J_{2}\right]\right](X, Y)= & J_{2}\left[\left[J_{2}, J_{2}\right]\right]\left(X, J_{1} Y\right)+J_{2}\left[\left[J_{2}, J_{2}\right]\right]\left(J_{1} X, Y\right)+ \\
& J_{3}\left[\left[J_{2}, J_{2}\right]\right]\left(J_{1} X, J_{1} Y\right)-J_{3}\left[\left[J_{2}, J_{2}\right]\right](X, Y) .
\end{aligned}
$$


From (2.5), the integrability of $I_{1}=J_{1}$ and $I_{2}$ and dividing by $\frac{a b}{2} \neq 0$ we obtain

$$
\begin{aligned}
-\frac{2 b}{a}\left[\left[J_{2}, J_{2}\right]\right](X, Y) \equiv- & J_{3}\left[\left[J_{2}, J_{2}\right]\right](X, Y)+J_{3}\left[\left[J_{2}, J_{2}\right]\right]\left(J_{1} X, J_{1} Y\right) \\
& +J_{2}\left[\left[J_{2}, J_{2}\right]\right]\left(J_{1} X, Y\right)+J_{2}\left[\left[J_{2}, J_{2}\right]\right]\left(X, J_{1} Y\right) .
\end{aligned}
$$

On the other hand if we change $(X, Y)$ with $\left(J_{2} X, J_{2} Y\right)$ we obtain

$$
\begin{aligned}
\frac{2 b}{a}\left[\left[J_{2}, J_{2}\right]\right](X, Y) \equiv & J_{3}\left[\left[J_{2}, J_{2}\right]\right](X, Y)-J_{3}\left[\left[J_{2}, J_{2}\right]\right]\left(J_{1} X, J_{1} Y\right) \\
& +J_{2}\left[\left[J_{2}, J_{2}\right]\right]\left(J_{1} X, Y\right)+J_{2}\left[\left[J_{2}, J_{2}\right]\right]\left(X, J_{1} Y\right)
\end{aligned}
$$

and by comparison we deduce that

$$
\left[\left[J_{2}, J_{2}\right]\right]\left(X, J_{1} Y\right)+\left[\left[J_{2}, J_{2}\right]\right]\left(J_{1} X, Y\right) \equiv 0,
$$

therefore

$$
\left[\left[J_{2}, J_{2}\right]\right]\left(J_{1} X, J_{1} Y\right)-\left[\left[J_{2}, J_{2}\right]\right](X, Y) \equiv 0,
$$

and we conclude that

$$
\left[\left[J_{2}, J_{2}\right]\right] \equiv 0 .
$$

This implies that $\left[\left[J_{3}, J_{3}\right]\right] \equiv 0$ by $(2.7)$ so that $T^{H} \equiv 0$ and therefore $T^{Q}=0$.

Recall that a hypercomplex structure on a manifold $M$ is defined by two anticommuting complex structures $I_{1}$ and $I_{2}$; in other words $I_{1}$ and $I_{2}$ form a 90-degree angle. As an application of what we just proved we now show that in fact it is enough to assume that the angle is constant. This result holds in any dimension and it was also proved independently by Gauduchon [G3].

Proposition 2.5. Let $\left(M^{4 n}, Q\right)$ be almost quaternionic with $n \geq 1$. Two compatible complex structures $I_{1}, I_{2}$ generate, over $\mathbb{R}$, a hypercomplex structure $H=\left(J_{\alpha}\right)$ if and only if the function $a=\left\langle I_{1}, I_{2}\right\rangle$ is a constant different from \pm 1 . In this case $\left(M^{4 n}, Q\right)$ is necessarily quaternionic or anti-self-dual when $n=1$.

Proof. By hypothesis $a$ and $b$ are constant; therefore the proof of Theorem 2.4 goes through globally on $M$ with each $\equiv$ sign replaced by equality. The conclusion in this case is that $J_{2}$ and $J_{3}$ are both integrable so that $\left(J_{\alpha}\right)$ is a globally defined hypercomplex structure on $M$.

Remark 2.6. An easy consequence of the above proposition is the known result that if $H=\left(J_{\alpha}\right)$ is almost hypercomplex with $J_{1}$ and $J_{2}$ integrable, then $J_{3}$ is also integrable. This also follows from $(2.7)$. $\triangle$

Remark 2.7. It is shown in [AGS] that the Iwasawa manifold $(M, g)$ of real dimension 6 admits an $S^{2}$-worth of Hermitian structures $\left(J_{\alpha}\right)_{\alpha \in S^{2}}$ such that $g\left(J_{\alpha}, J_{\beta}\right)=$ constant. However $M$ admits no hypercomplex structures for dimensional reasons. $\triangle$

\section{The TWISTOR SPACE OF AN ALMOST QUATERNIONIC MANIFOLD}

We show in this section that the twistor space $Z$ of an almost quaternionic manifold $\left(M^{4 n}, Q\right)$ admits a canonical almost complex structure $\mathbb{J}$. The idea is to apply the usual tautological twistor construction of Penrose to a restricted class of almost quaternionic connections called Oproiu connections (see $[\mathrm{AM}]$ and text below). Our construction generalizes the four-dimensional case considered in [AHS] as well as the quaternionic case of Salamon [S1], [S3] (see Remark 3.7.4). 
Given an almost quaternionic manifold $\left(M^{4 n}, Q\right)$, we consider the twistor fibration $t: Z \rightarrow M$ with total space $Z=\{q \in Q \mid\langle q, q\rangle=1\}$ and fiber $t^{-1}(p)$ equal the 2 -sphere of compatible almost complex structures on $T_{p} M$; for this reason a point of $Z$ will be usually denoted by $J$. Because $Z$ is a bundle of almost complex structures, any almost quaternionic connection $\nabla$ on $T M$ induces a connection also denoted by $\nabla$ in $Z \subset \operatorname{End}(T M)$ which can be used to define a 'tautological' almost complex structure $\mathbb{J}^{\nabla}$ on $Z$.

Our aim is to show that $Z$ carries a canonical almost complex structure $\mathbb{J}$ which depends only on $Q$ and not on a particular almost quaternionic connection $\nabla$.

For this purpose recall that an almost quaternionic connection $\nabla$ on $\left(M^{4 n}, Q\right)$ is called an Oproiu connection if its torsion tensor $T^{\nabla}=\operatorname{Tor}(\nabla)$ coincides with the structure tensor of $Q$ :

$$
T^{\nabla}=T^{Q} .
$$

Oproiu also defined $[\mathrm{O}]$ a projection which associates to any almost quaternionic connection $\nabla$ an Oproiu connection ${ }^{O p} \nabla$ so that any almost quaternionic manifold $\left(M^{4 n}, Q\right)$ admits many such connections.

The important fact is that any two Oproiu connections $\nabla$ and $\nabla^{\prime}$ on TM are related by

$$
\nabla^{\prime}=\nabla+S^{\xi}
$$

where $\xi \in \Lambda^{1} M$ is a 1 -form on $M$ and

$$
S_{X}^{\xi}=\xi(X) I d+X \otimes \xi-\sum_{\alpha} \xi\left(J_{\alpha} X\right) J_{\alpha}-\sum_{\alpha} J_{\alpha} X \otimes\left(\xi \circ J_{\alpha}\right)
$$

for any vector field $X$ and local admissible basis $\left(J_{\alpha}\right)$ on $M$. Therefore the induced connections on $Z \subset \operatorname{End}(T M)$ are related by

$$
\nabla^{\prime}=\nabla+S^{\xi}
$$

For an almost quaternionic connection $\nabla$ on $\left(M^{4 n}, Q\right)$, the Penrose almost complex structure $\mathbb{J}^{\nabla}$ on $Z$ is defined in the following way. At each point $J \in Z$ we split the tangent space

$$
T_{J} Z=V \oplus H^{\nabla}
$$

into the vertical space $V=\operatorname{Ker}\left(t_{*}\right)$ and the horizontal space $H^{\nabla}$ of the connection $\nabla$. For any compatible complex structure $J$ on $T_{p} M$ the vertical space $V_{J}$ is the space $\operatorname{Ker}\left(t_{*}\right)$ tangent to the fiber $t^{-1}(p)=S^{2}$ and is therefore given by those elements of $Q$ which are orthogonal to $J$ :

$$
V_{J}=\left\{q \in Q_{p} \mid\langle q, J\rangle=0\right\} .
$$

As usual the endomorphism $\mathbb{J}^{\nabla}$ is defined to preserve the splitting (3.2) by the formulas

$$
\mathbb{J}^{\nabla} q:=J \circ q, \quad \mathbb{J}^{\nabla} \tilde{X}:=\widetilde{J X}
$$

where $q \in V_{J}$ and $\tilde{X}, \widetilde{J X}$ are the $\nabla$-horizontal lifts of $X, J X \in T_{p} M$.

The remarkable fact is that the almost complex structure $\mathbb{J}^{\nabla}: T Z \rightarrow T Z$ does not depend on the Oproiu connection $\nabla$. Our proof is completely analogous to the argument of Gauduchon in the conformal Riemannian case [G1]. 
Theorem 3.1. For any $n \geq 2$ the twistor space $Z$ of an almost quaternionic manifold $\left(M^{4 n}, Q\right)$ admits a canonical almost complex structure $\mathbb{J}$. Furthemore, $(Z, \mathbb{J})$ is $a(2 n+1)$-dimensional complex manifold if and only if $\left(M^{4 n}, Q\right)$ is a quaternionic manifold-i.e. $T^{Q}=0$.

Proof. Let $\nabla$ and $\nabla^{\prime}$ be two Oproiu connections on $\left(M^{4 n}, Q\right)$. We want to show that the two tautological almost complex structures defined as above are equal: $\mathbb{J}^{\nabla}=\mathbb{J}^{\nabla^{\prime}}$. We will then set $\mathbb{J}=\mathbb{J}^{\nabla}$ for any Oproiu connection $\nabla$ on $\left(M^{4 n}, Q\right)$.

For any tangent vector $U \in T_{J} Z$ we will denote by $J_{1}(t)$ a curve in $Z$ extending $J$ and tangent to $U$-i.e. $J_{1}(0)=J$ and $J_{1}^{\prime}(0)=U$. Similarly, let $J_{2}(t)$ be the curve with $J_{2}(0)=J$ and $J_{2}^{\prime}(0)=\mathbb{J} U$ and let also $t_{*} U=X \in T_{p} M$. By the definition of covariant derivative, the $\nabla$-vertical component of $U$ is $\nabla_{X} J_{1}(t)$ and we will write the decomposition of $U$ as

$$
U=\left(\nabla_{X} J_{1}(t), X_{\nabla}\right) \in V \oplus H^{\nabla}
$$

where $X_{\nabla}$ is the horizontal lift of $X \in T_{p} M$. Similarly

$$
U=\left(\nabla_{X}^{\prime} J_{1}(t), X_{\nabla^{\prime}}\right) \in V \oplus H^{\nabla^{\prime}}
$$

From the definition of $\mathbb{J}^{\nabla}$, we obtain

$$
\mathbb{J}^{\nabla} U=\left(J \circ \nabla_{X} J_{1}(t),(J X)_{\nabla}\right)
$$

and from (3.3) we also have

$$
\mathbb{J}^{\nabla} U=\left(\nabla_{J X} J_{2}(t),(J X)_{\nabla}\right)
$$

and therefore

$$
\nabla_{J X} J_{2}(t)=J \circ \nabla_{X} J_{1}(t)
$$

Using (3.1) we have

$$
\mathbb{J}^{\nabla^{\prime}} U=\left(J \circ \nabla_{X}^{\prime} J_{1}(t),(J X)_{\nabla^{\prime}}\right)=\left(J \circ \nabla_{X} J_{1}(t)+J \circ\left[S_{X}^{\xi}, J\right],(J X)_{\nabla^{\prime}}\right)
$$

while

$$
\mathbb{J}^{\nabla} U=\left(\nabla_{J X}^{\prime} J_{2}(t),(J X)_{\nabla^{\prime}}\right)=\left(\nabla_{J X} J_{2}(t)+\left[S_{J X}^{\xi}, J\right],(J X)_{\nabla^{\prime}}\right) .
$$

To show that $\mathbb{J}^{\nabla}=\mathbb{J}^{\nabla^{\prime}}$ we use (3.4) and the identity

$$
J \circ\left[S_{X}^{\xi}, J\right]=\left[S_{J X}^{\xi}, J\right]
$$

which follows from the identity

$$
\left[S_{X}^{\xi}, J\right]=2 \xi\left(J_{2} X\right) J_{3}-2 \xi\left(J_{3} X\right) J_{2}
$$

valid for any admissible basis of $Q$ of the form $H=\left(J_{1}=J, J_{2}, J_{3}\right)$ (see [AM, $1.3 .2])$.

To investigate the integrability of $\mathbb{J}$ we compute the Nijenhuis tensor $N_{\mathbb{J}}$ using the formulas of Gauduchon [G1, p. 615] which are still valid for any almost quaternionic connection $\nabla$. In particular it follows from there that if $\tilde{X}$ and $\tilde{Y}$ are the $\nabla$ horizontal lifts in $T Z$ of vector fields $X, Y$ in $T M$, then the $\nabla$-horizontal component of $N_{\mathbb{J}}(\tilde{X}, \tilde{Y})$ is projected to $-T_{X}^{\nabla} Y$ by $t_{*}$. Since $\nabla$ is an Oproiu connection, the integrability of $\mathbb{J}$ implies the 1 -integrability of $Q$-i.e. $T^{Q}=0$. Vice versa it is well known that $(Z, \mathbb{J})$ is a complex manifold when $\left(M^{4 n}, Q\right)$ is quaternionic [S1], $[\mathrm{AG}]$. 
Before stating some of the properties of the almost complex manifold $(Z, \mathbb{J})$ in Proposition 3.6 we need some preliminary results. Let $\left(M^{4 n}, Q\right)$ be an almost quaternionic manifold with almost quaternionic connection $\nabla$. Then for any admissible basis $H=\left(J_{\alpha}\right)$ of $Q$ there are connection 1 -forms $\left(\omega_{\alpha}\right), \alpha=1,2,3$, with

$$
\nabla_{X} J_{\alpha}=\omega_{\gamma}(X) J_{\beta}-\omega_{\beta}(X) J_{\gamma}
$$

where $(\alpha, \beta, \gamma)$ is a cyclic permutation of $(1,2,3)$.

Lemma 3.2. Let $J$ be a compatible almost complex structure and let $\left(J=J_{1}, J_{2}, J_{3}\right)$ be an admissible basis of $\left(M^{4 n}, Q\right)$. Then

$$
\nabla_{J X} J=J \nabla_{X} J \quad \text { if and only if } \quad \omega_{2} \circ J_{2}=\omega_{3} \circ J_{3} .
$$

Proof. The conclusion follows immediately using (3.6):

$$
J\left(\nabla_{X} J\right)-\nabla_{J X} J=\left[\omega_{2}(X)-\omega_{3}\left(J_{1} X\right)\right] J_{2}+\left[\omega_{3}(X)+\omega_{2}\left(J_{1} X\right)\right] J_{3} .
$$

Definition 3.3. Let $H=\left(J_{\alpha}\right)$ be an admissible basis and $\nabla^{H}$ the associated Obata connection. Then we define the Oproiu connection of $H$ by

$$
{ }^{O p} \nabla^{H}=\nabla^{H}+\sum_{\alpha} \tau_{\alpha}^{H} \otimes J_{\alpha}
$$

where the structure 1 -forms $\tau_{\alpha}^{H}$ are defined in (2.3).

Remarks 3.4. 1) ${ }^{O p} \nabla^{H}$ is the Oproiu projection of the Obata connection $\nabla^{H}$.

2) The connection 1-forms of ${ }^{O p} \nabla^{H}$ are twice the structure 1-forms $\tau_{\alpha}^{H}$ :

$$
{ }^{O p} \nabla^{H} J_{\alpha}=2 \tau_{\gamma}^{H} \otimes J_{\beta}-2 \tau_{\beta}^{H} \otimes J_{\gamma} \text {. }
$$

$\triangle$

Proposition 3.5. For any admissible basis $H$ of $Q$ the structure 1-forms $\tau_{\alpha}^{H}$ and the connection 1-forms $\omega_{\alpha}$ of any Oproiu connection $\nabla$ are related by

$$
\left(\omega_{1}-2 \tau_{1}^{H}\right) \circ J_{1}=\left(\omega_{2}-2 \tau_{2}^{H}\right) \circ J_{2}=\left(\omega_{3}-2 \tau_{3}^{H}\right) \circ J_{3} .
$$

Proof. This is obvious for the Oproiu connection of $H$ and it follows for any Oproiu connection by (3.1), and (3.5).

We are now ready to prove the following result which gives conditions for a section of the twistor fibration $t: Z \rightarrow M$ to be a pseudo-holomorphic map and extends well known results in the quaternionic and conformal cases, see [S2], [G1], $[\mathrm{BdB}]$ for the latter case.

Proposition 3.6. Let $\left(M^{4 n}, Q\right)$ be an almost quaternionic manifold with twistor space $(Z, \mathbb{J})$. For a compatible almost complex structure $J$ on $M$ the following conditions are equivalent:

1. The image $J(M)$ is a $\mathbb{J}$-stable submanifold of $Z$.

2. $J:(M, J) \rightarrow(Z, \mathbb{J})$ is a pseudo-holomorphic map.

3. For any Oproiu connection $\nabla$ and any $X \in T M$

$$
\nabla_{J X} J=J \nabla_{X} J
$$

4. $J$ is parallel with respect to a (necessarily unique) Oproiu connection $\nabla^{J}$. Moreover when (1)-(4) hold, $J$ is integrable if and only if the $(0,2)$ part (with respect to $J$ ) of the torsion tensor $T^{Q}$ vanishes. 
Proof. To avoid confusion we use the notation $\sigma^{J}: M \rightarrow Z$ for the tautological section defined by $J$ and let $\sigma_{*}^{J}$ be its differential.

For any $X \in T M$ we have

$$
\sigma_{*}^{J}(J X)=\left(\nabla_{J X} \sigma^{J},(J X)_{\nabla}\right) \quad \text { and } \quad \mathbb{J}\left(\sigma_{*}^{J}(X)\right)=\left(J \nabla_{X} \sigma^{J},(J X)_{\nabla}\right) .
$$

Therefore (1),(2) and (3) are equivalent.

To show that (3) implies (4) we start from any Oproiu connection $\nabla$ satisfying Lemma 3.2. Then the new Oproiu connection $\nabla^{\prime}=\nabla+S^{\xi}$ where $\xi=-\frac{1}{2} \omega_{2} \circ J_{2}=$ $-\frac{1}{2} \omega_{3} \circ J_{3}$ preserves $J$, for any admissible basis $H=\left(J=J_{1}, J_{2}, J_{3}\right)$.

Finally, (4) implies (1) because the image of a parallel $J$ is horizontal and therefore $\mathbb{J}$-stable.

The last statement follows by applying [KN, prop. 3.6 (1)] to the Oproiu connection $\nabla^{J}$.

Remarks 3.7. 1) Our proof of Theorem 3.1 shows that two almost quaternionic connections $\nabla$ and $\nabla^{\prime}$ induce the same almost complex structure $\mathbb{J}^{\nabla}=\mathbb{J}^{\nabla^{\prime}}$ on $Z$ if and only if they have the same torsion: $T^{\nabla}=T^{\nabla^{\prime}}$. It also shows that the vanishing of the torsion $T^{\nabla}$ is a necessary condition for the integrability of $\mathbb{J}^{\nabla}$.

2) When $n=1$, an almost quaternionic structure is the same as an oriented conformal structure $[g]$ (see paragraph 5 below) and an Oproiu connection is a Weyl connection; in this dimension our construction coincides with the one in [AHS] and indeed our treatment is directly taken from Gauduchon [G1]. Notice that any Weyl connection $\nabla$ is torsion-free but in the conformal case the integrability of $\mathbb{J} \nabla$ is subject to a condition on the curvature $R^{\nabla}$, namely that the Weyl tensor $W$ of $[g]$ is anti-self-dual: $\star W=-W$. When $n>1$, the condition on the curvature $R^{\nabla}$ is automatically satisfied by any quaternionic connection [B, Proof of 14.74].

3) As it is well known in the conformal and quaternionic cases, the twistor space $Z$ satisfies the following naturality property. The group of almost quaternionic transformations of $\left(M^{4 n}, Q\right)$ is isomorphic to the group of pseudo-biholomorphisms of $(Z, \mathbb{J})$ which commute with the anti-pseudo-holomorphic involution $\tau: Z \rightarrow Z$ given by $\tau(J)=-J$.

4) As observed at the end of the proof of Theorem 3.1 Oproiu connections are torsion-free if and only if $Q$ is quaternionic; therefore in this particular case $(Z, \mathbb{J})$ is the twistor space defined by S. Salamon. Furthermore, as pointed out by the referee, the assumption that the twistor space $(Z, \mathbb{J})$ of an almost quaternionic manifold $(M, Q)$ is complex implies that $Q$ is quaternionic by an inverse construction of $[\mathrm{PP}]$. Another related reference is $[\mathrm{L}]$ where Salamon's construction is inverted for pseudo-quaternion Kähler manifolds. $\triangle$

\section{QUATERniOnIC MANIFOLDS}

In this section we will concentrate on the situation when $\left(M^{4 n}, Q\right)$ is a quaternionic manifold equipped with a quaternionic connection $\nabla$, that is a torsionless connection which preserves $Q$. We start with a necessary and sufficient condition for the integrability of a compatible almost complex structure. This condition is formally equal to the Riemannian case [G2, lemma 2] and holds for any $n \geq 1$.

Proposition 4.1. A compatible almost complex structure $J$ on a quaternionic manifold $\left(M^{4 n}, Q\right)$ is integrable if and only if the following identity holds for all $X \in T M$ 
and for one (and hence any) quaternionic connection $\nabla$ :

$$
\nabla_{J X} J=J \nabla_{X} J
$$

or equivalently if and only if, for any admissible basis $\left(J=J_{1}, J_{2}, J_{3}\right)$

$$
\omega_{2} \circ J_{2}=\omega_{3} \circ J_{3} .
$$

Proof. Recall first that the two conditions are equivalent by Lemma 3.2. The Nijenhuis tensor $N_{J}=-\frac{1}{8}[[J, J]]$ of $J$ can be written by means of the torsionless connection $\nabla$ as follows:

$$
N_{J}(X, Y)=\frac{1}{4}\left\{\left[J\left(\nabla_{X} J\right)(Y)-\left(\nabla_{J X} J\right)(Y)\right]-\left[J\left(\nabla_{Y} J\right)(X)-\left(\nabla_{J Y} J\right)(X)\right]\right\} .
$$

This proves one direction of the statement.

To prove in the other direction, let $\left(J=J_{1}, J_{2}, J_{3}\right)$ be an admissible basis; then from (3.6)

$$
\begin{aligned}
4 N_{J}(X, Y)= & {\left[\omega_{2}(X)-\omega_{3}(J X)\right] J_{2} Y+\left[\omega_{3}(X)+\omega_{2}(J X)\right] J_{3} Y } \\
& -\left[\omega_{2}(Y)-\omega_{3}(J Y)\right] J_{2} X-\left[\omega_{3}(Y)+\omega_{2}(J Y)\right] J_{3} X .
\end{aligned}
$$

This implies that for $n>1$ the Nijenhuis tensor $N_{J}=0$ if and only if $\omega_{2} \circ J_{2}-$ $\omega_{3} \circ J_{3}=0$ by choosing a vector $X$ which does not belong to the quaternionic line generated by $Y$. The same conclusion holds also for $n=1$ as the following formula shows :

$$
\begin{aligned}
4 N_{J_{1}}\left(X, J_{2} X\right)= & -\left[\omega_{2}(X)-\omega_{3}\left(J_{1} X\right)\right] X-\left[\omega_{3}(X)+\omega_{2}\left(J_{1} X\right)\right] J_{1} X \\
& -\left[\omega_{2}\left(J_{2} X\right)-\omega_{3}\left(J_{3} X\right)\right] J_{2} X-\left[\omega_{3}\left(J_{2} X\right)+\omega_{2}\left(J_{3} X\right)\right] J_{3} X
\end{aligned}
$$

We are now ready to prove the main result of the section which gives a precise relation between the local existence of compatible complex structures on quaternionic manifolds and twistor theory. It should be compared with the four-dimensional situation described in 5.8.

Theorem 4.2. For an almost quaternionic manifold $\left(M^{4 n}, Q\right)$ with $n \geq 2$ the following conditions are equivalent:

1. $\left(M^{4 n}, Q\right)$ is quaternionic.

2. The twistor space $(Z, \mathbb{I})$ is a complex $(2 n+1)$-manifold.

3. For any point $p \in M$ and any compatible complex structure $J$ at $T_{p} M$ there exist (infinitely many) integrable compatible complex structures which extend $J$ in a neighborhood of $p$.

4. In a neighborhood of any point $p \in M$ there exist two compatible complex structures $I_{1} \neq \pm I_{2}$.

Proof. By Theorem 2.4 we have that (4) implies (1) which in turns implies (2) by Theorem 3.1. When $(Z, \mathbb{J})$ is a complex manifold, for any point $J \in Z$ there are plenty of locally defined smooth complex hypersurfaces $D$ which contain $J$ and have intersection number 1 with the fibers of the twistor fibration which are holomorphically imbedded $\mathbb{C P}_{1}$ 's. But then any such $D$ can be thought of as a compatible complex structure extending $J$ in a neighborhood of $t(J)=p \in M$, by 3.6 and 4.1. This shows that (2) implies (3). 
Remarks 4.3. 1) In contrast to the local situation described above, there exist compact quaternionic manifolds $\left(M^{4 n}, Q\right)$ which do not admit globally defined compatible complex structures - e.g. compact quaternion-Kähler manifolds which are not locally hyper-Kähler. On the other hand there exist compact quaternionic manifolds which admit globally defined compatible complex structures and are not locally hypercomplex [P1].

2) The result of Theorem 4.2 cannot be improved. In 5.10 we will construct two examples, one local and the other compact, of almost quaternionic manifolds with a compatible complex structure which is unique even locally.

\section{FOUR-DIMENSIONAL MANIFOLDS}

In dimension four quaternionic geometry is the same as conformal geometry. More precisely a quaternionic structure $Q$ on an oriented manifold $\left(M^{4}\right.$, or $)$ is just the choice of a conformal class of Riemannian metrics $[g]$. In terms of $G$ structures this is simply the isomorphism $C O^{+}(4)=\mathbb{R}^{+} S O(4)=G L(1, \mathbb{H}) S p(1)$. Each representative $g \in[g]$ defines a $S O(4)=S p(1) S p(1)$-structure on $M^{4}$ and the quaternionic bundle $Q$ can be identified with the bundle of self-dual 2-forms $\Lambda_{+}^{2}-$ i.e. the 2-forms which are invariant under the Hodge star operator $\star: \Lambda^{2} \rightarrow \Lambda^{2}$. Notice that because $\star$ is conformally invariant on middle-dimensional forms, the bundle $Q \cong \Lambda_{+}^{2}$ is independent of $g \in[g]$ and the Levi-Civita connection of any $g \in[g]$ preserves $Q$. For these reasons throughout this section we will use the notation $Q=([g]$, or $)$.

An almost complex structure $J$ on $\left(M^{4},[g]\right.$, or $)$ is compatible with $Q$ if and only if $J$ induces the given orientation on $M^{4}$ and for all $X, Y \in T M$ we have $g(J X, J Y)=$ $g(X, Y)$ for some (and hence any) metric $g \in[g]$. If we fix a Riemannian metric $g \in[g]$, we can consider the Kähler form $F$ of $J$ which induces an isomorphism $F: \Lambda^{1} \rightarrow \Lambda^{3}$ so that there always exists $\theta \in \Lambda^{1}(M)$ satisfying $d F=\theta \wedge F$; the 1 -form $\theta$ is called the torsion form or Lee form of the almost Hermitian structure $(g, J)$ and it also satisfies $\theta=-\delta F \circ J$. It is easily seen that when $g$ is replaced by a conformally related metric $e^{f} g$, the Lee form $\theta$ is replaced by $\theta+d f$.

As in $\S 2$ we are interested in the situation when $\left(M^{4},[g]\right.$,or $)$ admits two compatible complex structures $I_{1} \neq \pm I_{2}$ and we will need the observation that when $J_{1}$ and $J_{2}$ are compatible with $[g]$ the difference of the Lee forms $\theta_{1}-\theta_{2}$ does not depend on the particular Riemannian metric $g \in[g]$.

The following formula for the differential of the angle function $a$ is due to Grantcharov [Gr]. Notice that both $a$ and $\theta_{1}-\theta_{2}$ are conformal invariants.

Proposition 5.1. Let $I_{1}$ and $I_{2}$ be compatible complex structures on $\left(M^{4},[g]\right.$, or $)$. Then

$$
d a=2\left(\theta_{1}-\theta_{2}\right) \circ\left[I_{1}, I_{2}\right] .
$$

Proof. Let $\nabla$ be the Levi-Civita connection of $g$. From Remark 2.1 (5) we have

$$
\nabla_{X} a=\nabla_{X}\left(\sum_{r=1}^{4} g\left(I_{1} E_{r}, I_{2} E_{r}\right)\right)=\sum_{r=1}^{4}\left(g\left(\nabla_{X}\left(I_{1} E_{r}\right), I_{2} E_{r}\right)+g\left(I_{1} E_{r}, \nabla_{X}\left(I_{2} E_{r}\right)\right)\right) .
$$

Because $I_{1}$ is integrable a well known formula [KN, p.148] implies that

$$
g\left(\nabla_{X}\left(I_{1} E_{r}\right), I_{2} E_{r}\right)=d F_{1}\left(X, I_{1} E_{r}, I_{1} I_{2} E_{r}\right)-d F_{1}\left(X, E_{r}, I_{2} E_{r}\right)
$$


so that

$$
\begin{aligned}
& \sum_{r=1}^{4}\left(g\left(\nabla_{X}\left(I_{1} E_{r}\right), I_{2} E_{r}\right)\right) \\
& =\sum_{r=1}^{4}\left(\theta_{1}(X) F_{1}\left(E_{r}, I_{2} E_{r}\right)-\theta_{1}\left(I_{1} E_{r}\right) F_{1}\left(X, I_{1} I_{2} E_{r}\right)+\theta_{1}\left(I_{1} I_{2} E_{r}\right) F_{1}\left(X, I_{1} E_{r}\right)\right. \\
& \left.-\theta_{1}(X) F_{1}\left(E_{r}, I_{2} E_{r}\right)+\theta_{1}\left(E_{r}\right) F_{1}\left(X, I_{2} E_{r}\right)-\theta_{1}\left(I_{2} E_{r}\right) F_{1}\left(X, E_{r}\right)\right) \\
& =2\left(\theta_{1}\left(I_{1} I_{2} X\right)-\theta_{1}\left(I_{2} I_{1} X\right)\right)=2 \theta_{1} \circ\left[I_{1}, I_{2}\right](X) .
\end{aligned}
$$

Similarly for $I_{2}$ and the proposition follows.

Corollary 5.2. [Bo] Two compatible complex structures $I_{1} \neq \pm I_{2}$ on $\left(M^{4},[g]\right.$, or $)$ generate a hypercomplex structure (over $\mathbb{R}$ ) if and only if they have the same Lee form.

The main result of this section is the following 'maximum principle'.

Theorem 5.3. Suppose that $\left(M^{4},[g]\right.$, or $)$ admits two compatible complex structures $I_{1} \neq \pm I_{2}$ and let $p \in M^{4}$ be a local extremum of the angle function a with $a(p) \neq \pm 1$. If the 1-form $\theta_{1}-\theta_{2}$ is closed, then all covariant derivatives of a must vanish at p. If furthermore $I_{1}, I_{2}$ induce the same real analytic structure or if the 1-form $\theta_{1}-\theta_{2}$ is harmonic with respect to some $g \in[g]$, then a must be constant.

Proof. Since $a(p) \neq \pm 1$, in a neighborhood of $p$, we can set $a=\cos \alpha$ and define a new complex structure $K$ by $K=\frac{1}{2}\left(1-a^{2}\right)^{-\frac{1}{2}}\left[I_{1}, I_{2}\right]=\frac{1}{2 \sin \alpha}\left[I_{1}, I_{2}\right]$ so that $d \alpha=4\left(\theta_{2}-\theta_{1}\right) \circ K$. Since $\theta_{1}-\theta_{2}$ is closed, we can write

$$
d \alpha=d l \circ K
$$

for some function $l$ defined in a neighborhood of $p$. Notice that $p$ is also a local extremum of $\alpha$.

The proof of the theorem rests on the following two lemmas of multilinear algebra. To simplify the notation we will denote by $A_{m}$ the $m$-th covariant derivative of $\alpha$ at $p$; similarly $L_{m}$ will denote the $m$-th covariant derivative of $l$ at $p$ so that $A_{m}$ and $L_{m}$ are elements of $\otimes^{m}\left(T_{p}^{*} M\right)$ and (5.1) can be written as $A_{1}=L_{1} \circ K$. The first lemma tells us that similar relations hold for higher order derivatives:

Lemma 5.4. Under the hypothesis of Theorem 5.3 suppose also that the covariant derivatives of a at $p$ vanish up to order $n-1$. Then, for all $v_{1}, \ldots, v_{m} \in T^{*} M$ and for all $m \leq n$ we have that

$$
A_{m}\left(v_{1}, \ldots, v_{m-1}, v_{m}\right)=L_{m}\left(v_{1}, \ldots, v_{m-1}, K v_{m}\right) .
$$

Proof. By induction on $n$. When $n=2$ equation (5.1) implies that $\nabla l=\nabla \alpha=0$ at $p$ so that $\nabla^{2} \alpha=\nabla^{2} l \circ K$ at $p$, as wanted. Similarly, $\nabla^{n} \alpha=\nabla^{n} l \circ K$ at $p$, because by induction $\nabla^{m} \alpha=\nabla^{m} l=0$ at $p$ for all $m \leq n-1$ since $b(p) \neq 0$; the lemma is now proved.

Denote by $V$ the complexification $\mathbb{C} \otimes T_{p}^{*} M$ of the cotangent space of $M$ at $p$ and consider the decomposition $V=V^{1,0} \oplus V^{0,1}$ into sum of $( \pm i)$-eigenspaces of the almost complex structure $K$.

We know that the first covariant derivatives of both $\alpha$ and $l$ vanish at $p$. Assume now that there exist the least integer $m \geq 2$ such that $A_{m} \neq 0$. By Lemma 5.4 
we have that (5.2) holds for any complex cotangent vectors $v_{1}, \ldots, v_{m} \in V$ and for short we will use the notation $A_{m}=L_{m} \circ K$.

Furthermore since $A_{n}=L_{n}=0$ for $n<m A_{m}$ and $L_{m}$ are symmetric tensors. We may consider them as elements of the $m$-th symmetric power $S^{m} V$ of $V$.

The almost complex structure $K$ induces a decomposition of every tensor space over $V$ into types - e.g. $S^{m} V=\bigoplus_{p+q=m} S^{p, q} V$. As a consequence of Lemma 5.4 we have the following result:

Lemma 5.5. Let $p \in M$ be a critical point of a with $a(p) \neq \pm 1$ and let $m \geq 2$ be the least integer such that $A_{m} \neq 0$. Then $A_{m} \in S^{m, 0} V \oplus S^{0, m} V$.

Proof. Let $\left\{e_{r}\right\}_{r=1, \ldots, 4}$ be a basis of $V^{1,0}$ and $\left\{\bar{e}_{r}\right\}_{r=1, \ldots, 4}$ be a basis of $V^{0,1}$; we can write $L_{m}=\sum_{r} M_{r} \otimes e_{r}+\sum_{r} N_{r} \otimes \bar{e}_{r}$ where each $M_{r}$ and $N_{r}$ are $(m-1)$-tensors; and we also have

$$
A_{m}=L_{m} \circ K=i \sum_{r} M_{r} \otimes e_{r}-i \sum_{r} N_{r} \otimes \bar{e}_{r} .
$$

Since $A_{m}$ and $L_{m}$ are both symmetric, we have that $L_{m}-i A_{m}=2 \sum_{r} M_{r} \otimes e_{r}$ is also symmetric; this forces $M_{r}$ to be of type $(m-1,0)$ for each $r$. Similarly $L_{m}+i A_{m}$ symmetric implies that $N_{r}$ is of type $(0, m-1)$ for each $r$ and therefore the decomposition of $A_{m}$ into types is $A_{m}=A^{m, 0}+A^{0, m}$ and the lemma is proved.

It is now easy to finish the proof of Theorem 5.3: since $A_{m}$ is a real tensor we also have that $A^{0, m}=\overline{A^{m, 0}}$. Now let $X=v+\bar{v} \in T_{p} M$ be a real tangent vector and compute $A_{m}(X, \ldots, X)=A_{m}(v+\bar{v}, \ldots, v+\bar{v})=A^{m, 0}(v, \ldots, v)+$ $A^{0, m}(\bar{v}, \ldots, \bar{v})=A^{m, 0}(v, \ldots, v)+\overline{A^{m, 0}}(\bar{v}, \ldots, \bar{v})=2 \operatorname{Re} A^{m, 0}(v, \ldots, v)$. Next we choose a complex number $\lambda$ with $\lambda^{m}=-1$ and consider the real vector $Y=\lambda v+\overline{\lambda v}$; then $A_{m}(Y, \ldots, Y)=2 \lambda^{m} \operatorname{Re} A^{m, 0}(v, \ldots, v)=-A_{m}(X, \ldots, X)$ which shows that $A_{m}$ cannot be semidefinite unless it is zero. The first part of the theorem is proved.

Assume now that $I_{1}, I_{2}$ define the same real analytic structure on $M$. Then the angle function $a=-\frac{1}{4} \operatorname{tr}\left(I_{1} I_{2}\right)$ is real analytic; hence it is constant if it is flat in one point. Similarly, if $\theta_{1}-\theta_{2}$ is harmonic, the same holds for the locally defined function $l$ which therefore has to be constant if it is flat at one point.

A nice application of the above theorem is the following result which was used in the classification of compact anti-self-dual manifolds with two compatible complex structures [P2]; recall that the conformal Weyl tensor $W$ of an oriented four-manifold with conformal structure $[g]$ decomposes into $W=W_{+}+W_{-}$with $\star\left(W_{ \pm}\right)= \pm W_{ \pm}$. The manifold $\left(M^{4},[g]\right.$, or $)$ is said to be anti-self-dual if and only if $W_{+}=0$; this notion is important also because it provides a useful link to complex geometry given by the fact that the Penrose twistor space $Z$ of an anti-selfdual manifold is a complex three-dimensional manifold [AHS].

Corollary 5.6. Let $\left(M^{4},[g]\right.$, or $)$ be a compact anti-self-dual four-manifold with two compatible complex structures $I_{1} \neq \pm I_{2}$. If the function $a: M \rightarrow[-1,1]$ is not constant, then it must be surjective and the preimages $a^{-1}( \pm 1)$ are two disjoint curves which are complex with respect to both $I_{1}$ and $I_{2}$.

Proof. In this case it is shown in [P2] that $\theta_{1}-\theta_{2}$ is harmonic for some $g \in[g]$. If $a$ is not onto, there exists a global extremum $p \in M$ with $a(p) \neq \pm 1$ and the result follows from Theorem 5.3. Furthermore $a^{-1}( \pm 1)$ are complex curves because they 
can be thought as the intersection of the complex hypersurfaces $I_{1}(M)$ and $\pm I_{2}(M)$ in the complex 3-manifold $Z$ which is the twistor space of $\left(M^{4},[g]\right.$,or $)$ [P2]. Here we consider a complex structure $I$ as a section $I: M \rightarrow Z$.

Remark 5.7. It was communicated to us by Gauduchon that $d\left(\theta_{1}-\theta_{2}\right)=0$ implies that $([g]$,or $)$ is anti-self-dual. $\triangle$

The following statement describes results of various authors [AHS] [S4] [K], see also [P2] by relating twistor theory, anti-self-duality and the existence of compatible complex structures in four-dimensional Riemannian geometry. It should be compared with Theorem 4.2.

Theorem 5.8. On an oriented Riemannian conformal four-manifold $\left(M^{4},[g]\right.$, or $)$ the following conditions are equivalent:

1. $\left(M^{4},[g]\right.$, or $)$ is anti-self-dual.

2. The twistor space $(Z, \mathbb{J})$ is a complex 3-manifold.

3. For any point $p \in M$ and any compatible complex structure $J$ at $T_{p} M$ there exist (infinitely many) integrable compatible complex structures which extend $J$ in a neighborhood of $p$.

4. In a neighborhood of any point $p \in M$ there exist three compatible complex structures $I_{1}, I_{2}$ and $I_{3}$ with $I_{i} \neq \pm I_{j}$ for $i \neq j$.

Proof. The twistor space of $\left(M^{4},[g], o r\right)$ and the equivalence between (1) and (2) were described in [AHS] following ideas of Penrose. That (2) implies (3) is a standard argument which can also be found in our proof of 3.2. Obviously (3) implies (4). Finally, Salamon proved that (4) implies (1) [S4, p. 121]; see also [P2].

Remark 5.9. It was shown by Kobak $[\mathrm{K}]$ that there are explicit examples of oriented Riemannian four-manifolds, even compact, with exactly two compatible complex structures. Therefore the result of 5.8 cannot be improved. $\Delta$

The following examples show that Theorem 4.2 cannot improved either.

Examples 5.10. 1) Let $\left(M^{4}, g, J\right)$ be any Hermitian surface which is not antiself-dual - e.g. any Kähler surface of scalar curvature not identically zero, and let $Q \cong \Lambda_{+}^{2}$ be the almost quaternionic structure defined by the metric $g$ and the orientation induced by $J$. Let $H=\left(J=J_{1}, J_{2}, J_{3}\right)$ be an admissible basis of $Q$ and assume that $H$ is defined everywhere on $M$. Let $M^{\prime}$ be the product $M \times M$ equipped with the product almost hypercomplex structure $H^{\prime}=\left(J^{\prime}=J_{1}^{\prime}, J_{2}^{\prime}, J_{3}^{\prime}\right)$ with $J_{\alpha}^{\prime}=J_{\alpha} \times J_{\alpha}$ for $\alpha=1,2,3$ and notice that $J^{\prime}$ is a complex structure.

Now let $(Z, \mathbb{J})$ and $\left(Z^{\prime}, \mathbb{J}^{\prime}\right)$ denote the twistor spaces of $(M, H)$ and $\left(M^{\prime}, H^{\prime}\right)$ respectively. We want to show that $J^{\prime}$ is the unique compatible complex structure of $H^{\prime}$. By Theorem 4.2 it is enough to prove that $T^{Q^{\prime}} \neq 0$ where $Q^{\prime}=\left\langle H^{\prime}\right\rangle$ and this is equivalent to showing that $\left(Z^{\prime}, \mathbb{J}^{\prime}\right)$ is not a complex manifold. The diagonal imbedding $d: M \hookrightarrow M^{\prime}$ is quaternionic and therefore the induced map $\tilde{d}:(Z, \mathbb{J}) \hookrightarrow\left(Z^{\prime}, \mathbb{J}^{\prime}\right)$ is pseudo-holomorphic. If we now assume by contradiction, that $\left(Z^{\prime}, \mathbb{J}^{\prime}\right)$ is a complex manifold, then $(Z, \mathbb{J})$ would also be complex. This contrasts with the assumption that $g$ is not anti-self-dual and we conclude that $J^{\prime}$ is unique. 
2) To construct a compact example one can take $\left(M^{4}, g, J\right)$ to be the 'KodairaThurston' four-manifold considered by Salamon in [S4]. $\Delta$

\section{ACKNOWLEDGEMENTS}

The last two authors would like to thank Paul Gauduchon for the course on the Riemannian Golberg-Sachs theorem he gave in Rome at the Department of Mathematics "G. Castelnuovo" on October 1996 and which inspired to us some of the results of $\S 3$.

\section{REFERENCES}

[AGS] E. Abbena, S. Garbiero, S. Salamon, Hermitian geometry on the Iwasawa manifold, Preprint 1995.

[AG] D.V. Alekseevsky, M.M. Graev, G-structures of twistor type and their twistor spaces, J. Geom. Phys. 3 (1993), 203-229. MR 94e:53026

[AHS] M.F. Atiyah, N.J. Hitchin, I.M. Singer, Self-duality in four-dimensional Riemannian geometry, Proc. Roy. Soc. London A 362 (1978), 425-461. MR 80d:53023

[AM] D.V. Alekseevsky, S. Marchiafava, Quaternionic structures on a manifold and subordinated structures, Annali di Mat. Pura e Appl. (4) 171 (1996), 205-273. CMP 97:10

[B] A. Besse, Einstein manifolds, Ergebnisse der Math. 3 Folge Band 10, Springer, Berlin New York, 1987. MR 88f:53087

[Bo] C. Boyer, A note on hyperHermitian four-manifolds, Proc. Amer. Math. Soc. 102 (1988), 157-164. MR 89c:53049

[BdB] D. Burns, P. de Bartolomeis, Applications harmoniques stables dans $\mathbb{P}^{n}$, Ann. Sci. École Norm. Sup. (4) 21 (1988), 159-177. MR 89h:58044

[G1] P. Gauduchon, Structures de Weyl et théorèmes d'annulation sur une variété conforme autoduale, Ann. Scuola Norm. Sup. Pisa Cl. Sci. 18 (1981), 563-629. MR 93d:32046

[G2] - Complex structures on compact conformal manifolds of negative type, Complex Analysis and Geometry (V. Ancona, E. Ballico, S. Silva, eds.), Proceedings of the conference at Trento, Marcel Dekker, New York, Basel, Hong Kong, 1996, pp. 201-212. MR 96m:53079

[G3] Canonical connections for almost-hypercomplex structures, Pitman Res. Notes in Math. Ser., Longman, Harlow, 1997. CMP 98:03

[Gr] G. Grantcharov, Private communications.

[K] P. Kobak, Explicit doubly-Hermitian metrics, ESI preprint (1995).

[KN] S. Kobayashi , K. Nomizu, Foundations of differential geometry. II, Wiley, New York, 1969. MR 38:6501

[L] C. LeBrun, Quaternion Kähler manifolds and conformal geometry, Math. Ann. 284 (1989), 353-376. MR 90e:53062

[O] V. Oproiu, Integrability of almost quaternal structures, An. st. Univ. "Al. I. Cuza" Iasi 30 (1984), 75-84. MR 86k:53055

[P1] M. Pontecorvo, Complex structures on quaternionic manifolds, Diff. Geometry and its Applications 4 (1992), 163-177. MR 95b:53058

[P2] Complex structures on Riemannian 4-manifolds, Math. Ann. 309 (1997), 159-177. CMP 97:17

[PP] H. Pedersen, Y.S. Poon, Twistorial Construction of Quaternionic Manifolds, Proc. VIth Int. Coll. on Diff. Geom., Cursos y Congresos 61, 1995, pp. 207-218. MR 91f:53040

[S1] S. Salamon, Quaternionic manifolds, Symposia Mathematica (Rome, 1980), vol. XXVI, Academic Press, London - New York, 1982, pp. 139-151. MR 84e:53044

[S2] - Harmonic and holomorphic maps, Lecture Notes in Mathematics 1164 (E. Vesentini, eds.), Geometry Seminar Luigi Bianchi' II - 1984, Springer, Berlin Heidelberg New York, 1985, pp. 162-224. MR 88b:58039

[S3] Differential geometry of quaternionic manifolds, Ann. Sci. École Norm. Sup. (4) 19 (1986), 31-55. MR 87m:53079

[S4] _ Special structures on four-manifolds, Riv. Mat. Univ. Parma (4) 17 (1991), 109123. MR 94k:53064 
[T1] F. Tricerri, Sulle varietà dotate di due strutture quasi complesse linearmente indipendenti, Riv. Mat. Univ. Parma 3 (1974), 349-358. MR 55:4034

[T2] - Connessioni lineari e metriche Hermitiane sopra varietà dotate di due strutture quasi complesse, Riv. Mat. Univ. Parma 4 (1975), 177-186. MR 56:1241

Gen. Antonova 2, kv. 99, 117279 Moscow, Russian Federation

Current address: E. Schrödinger Institute, Bolzmanngasse 9, A-1090, Vienna, Austria

E-mail address: daleksee@esi.ac.at

Dipartimento di Matematica, Università di Roma "La Sapienza", P.le A. Moro 2, 00185 Roma, ITALY

E-mail address: marchiafava@axrma.uniroma1.it

Dipartimento di Matematica, Università di Roma Tre, L.go S.L. Murialdo 1, 00146 Roma, ITALY

E-mail address: max@matrm3.mat.uniroma3.it 